\title{
Catalogue of Tsunamis in the Eastern Mediterranean from Antiquity to Present Times
}

\author{
J. Antonopoulos
}

Received on June 14th, 1979

\begin{abstract}
This paper presents a systematic compilation of all data pertaining to tsunamis observed or recorded in the Eastern Mediterranean from antiquity to present times (1500 b.C. to 1980 a.D.). The first catalogue has been published by Prof. N.N. Ambraseys in the "Bulletin of the Seismological Society of America" (Vol. 52, No 4, October 1962) and this has formed the skeleton of this paper. All of the available information has been compiled from historical accounts, newspaper archives, other reports, and recent mareographic data. The earthquake data have been extracted from the Seismological Institute of Athens and relative bibliography. Most of the events listed are associated with earthquakes, but some are the result of volcanic activity. Many of the tsunamis have been generated along the coast of Greece, Albania, South Yugoslavia, Turkey, Syria and Israel. There is little doubt that the large conjugate fault system along, the west coast of Greece, Crete and Rodos till the south coast of Turkey is often responsible for the earthquakes in this area and most of tsunamis in the Eastern Mediterranean.
\end{abstract}




\section{RIASSUNTO}

In questo lavoro è stata fatta una raccolta sistematica di tutti i dati relativi agli tsunamis osservati o registrati nel Mediterraneo orientale dall'antichità ad oggi (1500 a.C. al 1980 d.C.). II primo catalogo è stato pubblicato dal Prof. N.N. Ambraseys nel "Bollettino della Società Americana" (Vol. 52, n. 4, ottobre 1962), sulla ossatura di quest'ultimo è stato costruito l'attuale lavoro. Sono state raccolte tut te le informazioni utili da resoconti storici, archivi di giornali, altre notizie, e dati mareografici recenti. I dati relativi ai terremoti sono stati estratti da quelli forniti dall'Istituto Sismologico di Atene con la relativa bibliografia. La maggior parte degli eventi in clenco, è associata a terremoti, mentre alcuni sono il risultato di attività vulcanica. Molti degli tsunamis hanno avuto origine lungo le coste della Grecia, Albania, Jugoslavia meridionale, Turchia, Siria ed Israele. Si ipotizza che il grande sistema di faglie coniugate, la costa occidentale della Grecia, Creta e Rodi fino alla costa meridionale della Turchia, sia spesso responsabile dei terremoti in questa zona e della maggior parte degli tsunamis nel Mediterraneo orientale.

\section{INTRODUCTION}

between $31^{\circ}-44^{\circ} \mathrm{N}$ and $18^{\circ}-36^{\circ} \mathrm{E}$ excluding Black Sea and the Italian coasts of the Adriatic Sea is the object of the present paper.

The first catalogue has published by N. Ambraseys in "Bulletin of the Seismological Society of America" (Vol. 52, No. 4, p.p. 895-913, October 1962) and this has formed the skeleton of this paper.

In this catalogue we have compiled and thus rendered available the mass of interesting information about seismic sea-waves which lies buried in many Greek, Byzantine, Arabic and Latin textes. As will be seen from the references, the material, both printed and manuscript, are ample. We are convinced that a systematic and scholarly investigation into so many of the manuscripts and textes which we were unqualified to render and which we left out, could easily double the entries and clarify many points in our catalogue.

Some feu of the Byzantine and Arabic sources we quote 
have, it is true, been read in whole or in part by few authors of earthquake catalogues; but as far as we are aware, for one reason or another, practically none of the information supplied in these catalogues is absolutely correct or complete. Some authors erred in rendering Muslim or Byzantine chronologies with the result to date in their catalogues scores of events by as much as six centuries too early (Ambraseys, 1961). Others, owing to errors resulting from transcribing events and toponymics from Latin translations of Greek or Arabic texts, or owing to differences in chronology resulting thereof, repeat in their catalogues the same event two or more times, or enter non-existant events. In some instances also they treat the seismicity of a whole year for a given region collectively and enter it as one earthquake. The most critical of these errors where they affect our catalogue are pointed out in their appropriate place in the paper.

Actual harm has been done to the science of seismology by premature conclusions from fragmentary data, and particularly from data repeated from publication to publication with a constantly increasing number of inaccuracies with no reference to the original source of information. Under such circumstances one can hardly be unjust to those who maintain that the observations of seismic phenomena of old date are scientifically inadequate.

In preparing our catalogue we were fully aware that its outcome would be more suggestive than definitive. Also we were aware of the fact that the older historical accounts of seismic phenomena rarely include much useful information other than reports of damage and casualties. These we considered worthwhile our labours.

\section{Key to catalogue}

The catalogue is arranged in the following manner.

Dates are given in headlines in the new style (Gregorian). They are followed by the name of the region in which the seismic sea-wave was felt. This is followed by the name of cities, towns 
or areas which were particularly affected. The intensity, $m$, of the wave is given in small roman numerals and it refers to Sieberg's modified intensity scale where the height of the wave on land or the distance which it flooded inland is known and is inticated as $(H=)$ and $(L=)$ respectively in meters. Near the geographical coordinates of the epicentre of the main earthquake shock, when known are given together with the intensity of the shock ( $I=$ in the modified Mercalli scale), magnitude ( $M=$ after Gutenberg) and the focal depth $(d=, s$ : shallow, $n$ :normal, $i$ intermediate). This is followed by the references consulted which are pertinent to the Phenomenon itself, to the accompanying earthquake, and to the information necessary for the dating of the event. Numerals outside the parentheses refer to references in the Bibliography. In the parentheses, roman numerals refer to the book (liber) and the following numbers to the chapter and line respectively of the text. P : refers to a standard system of pagination while $\mathrm{p}$ : indicates the page. Folio is indicated by $f$. Page references to an authority is not given when the material in that authority is arranged chronologically. Entries marked with an asterisk are doubtful events quoted mostly by modern writers, which have been included for the sake of completeness.

Place names mentioned in the original suffered many changes during historical and recent times. When these differ from those of today, the former are shown in brackets where they first occur, and the modern name is used thereafter. Modern places are spelt after Lippincott.

In what follows the headlines more information is given mainly about the events from $1500 \mathrm{BC}$ to $1650 \mathrm{AD}$ and some of them up to the present time. For all the other events only a bare outline is given.

With so many dates, controversial points, so many foreign names and such a multitude of references as crowd this paper; though we have done our best to be as accurate as possible, many errors must necessarily have crept in. We shall feel most greateful to those who will point these out to us. 


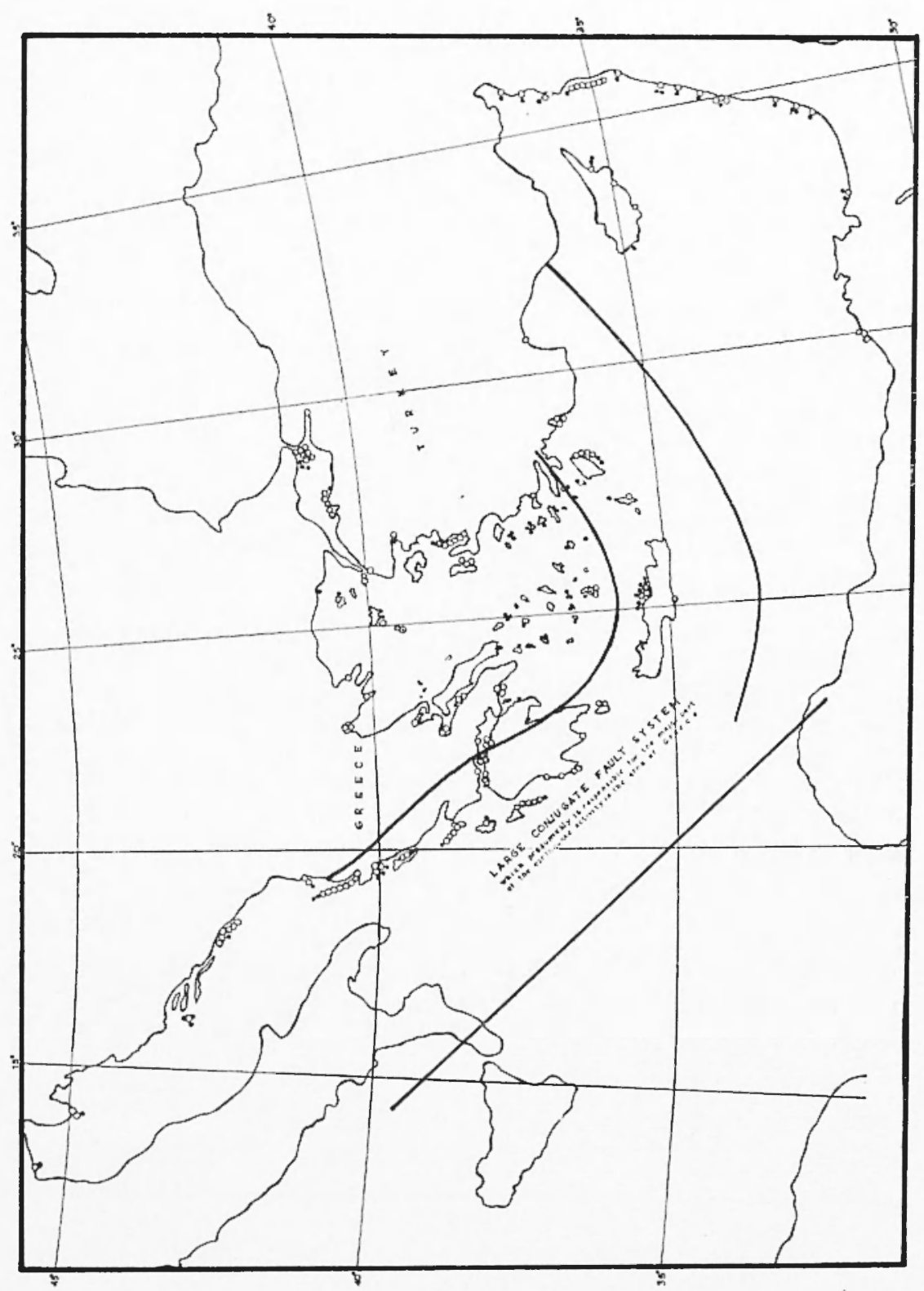

Fig. 1 - Tsunamis in the Eastern Mediterranean from 1500 BC to 1980 A.D. 


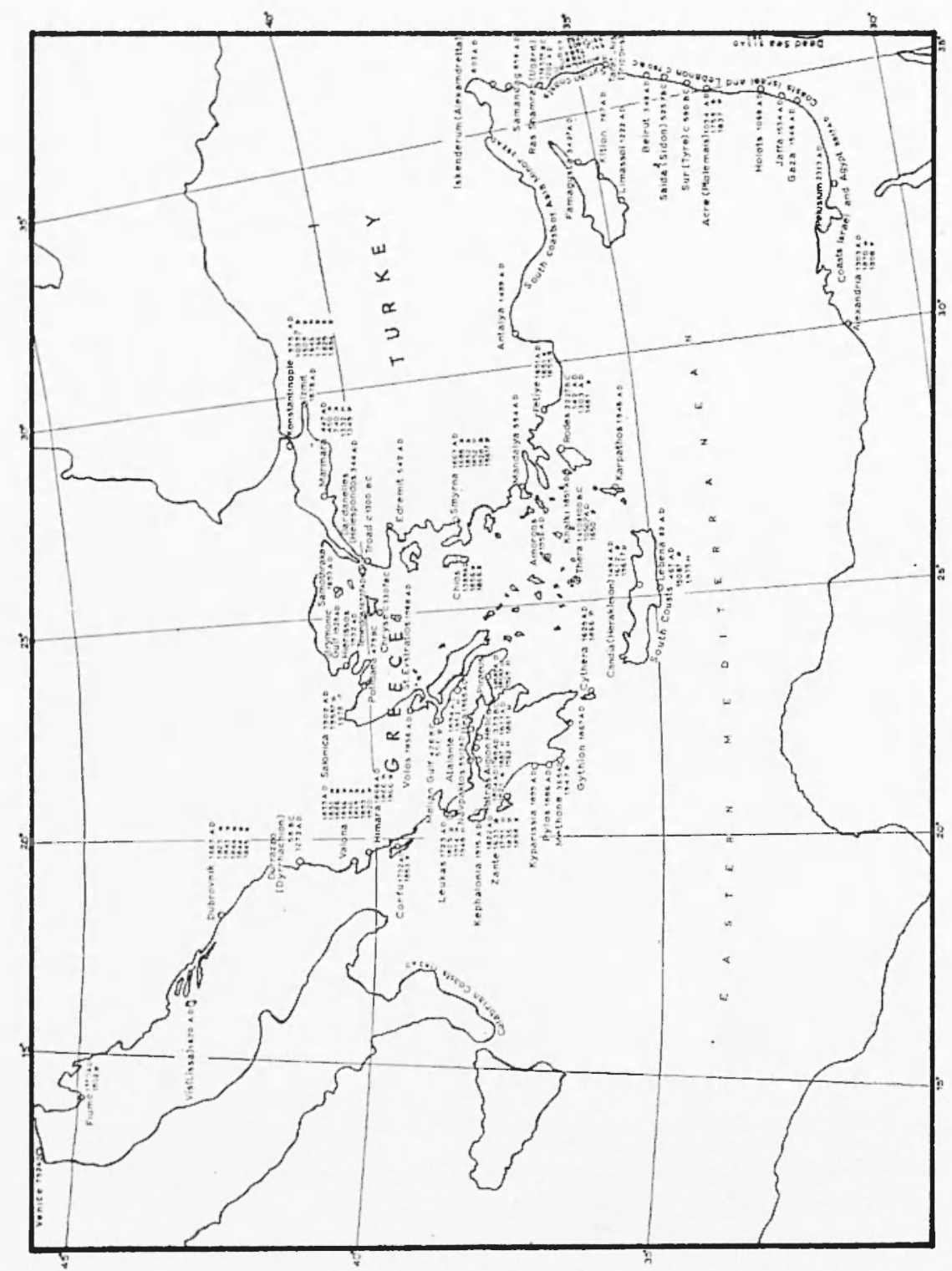

Fig. 2 - o = sources of tsunamis;

$o=$ region in which the tsunamis was felt. 


\section{A T A L O G E}

\begin{tabular}{|c|c|c|c|c|c|c|c|c|c|c|}
\hline \multirow{2}{*}{$\mathrm{N}^{\circ}$} & \multirow{2}{*}{ DATE } & \multicolumn{4}{|l|}{ TSUNAMI DATA } & \multicolumn{4}{|c|}{ EARTHQUAKE DATA } & \multirow{2}{*}{ REFERENCES } \\
\hline & & $\begin{array}{l}\text { Area of origin } \\
\text { observation place of }\end{array}$ & $m$ & $H$ & $L$ & $M$ & $I$ & $s, n, i$ & $\begin{array}{l}\text { Latitude } \\
\text { Longitude }\end{array}$ & \\
\hline 1. & II millenium B.C. & Syrian coasts & vI & & & & $\mathrm{X}$ & & $36^{\circ} 1 / 2 \mathrm{~N}-25^{\circ} 1 / 2 \mathrm{E}$ & 13. \\
\hline 2. & $1410 \pm 100$ & $\begin{array}{l}\text { North coast of Crete. Grecian } \\
\text { Archipelago }\end{array}$ & VI & & & & XII & & & $13,23,25,34,35$ \\
\hline 3. & $1365 \pm 5$ & $\begin{array}{l}\text { Syrian coasts. Ugarit near Minet- } \\
\text { el-Beida }\end{array}$ & $\mathrm{VI}$ & & & & & & & 13. \\
\hline 4. & c. 1300 & $\begin{array}{l}\text { Ionian coasts and towns in Asia } \\
\text { Minor, Troad }\end{array}$ & v & & & & & & & 13. \\
\hline 5. & c. 760 & Coasts of Israel, and Lebanon & & & & & & & & 13. \\
\hline 6. & c. 590 & Coasts of Lebanon. Sur (Tyre) & vI & & & & & & & $13,38$. \\
\hline 7. & c. $525^{*}$ & Coasts of Lebanon. Saida (Sidon) & & & & & & & & $5,6,13,38,39$ \\
\hline 8. & 479 Winter & $\begin{array}{l}\text { Chalcidice. Isthmus of Kassandra, } \\
\text { Potidaea }\end{array}$ & III & & & & IX & & $40^{01} 1,4 \mathrm{~N}-23^{\circ} 1 / 2 \mathrm{E}$ & 2,13 \\
\hline 9. & 426 Summer & $\begin{array}{l}\text { Euboean and Malian Gulfs, Phthio- } \\
\text { tian coasts, Euboean coasts, Is- } \\
\text { land of Skopelos }\end{array}$ & $v-$ & & & & $\mathrm{X}$ & & $38^{03 / 4} \mathrm{~N}-22^{03 / 4} \mathrm{E}$ & $6,7,13$ \\
\hline 10. & 373 Winter & Gulf of Corinth. Helice & $\mathrm{v}$ & & & & XI & & $38^{\circ} 1 / 4 \mathrm{~N}-22^{\circ} 1 / 4 \mathrm{E}$ & $1 \alpha, 1 \beta, 3,5,6,13$ \\
\hline 11. & c. $330^{*}$ & $\begin{array}{l}\text { Eastern Sporades Islands. Island } \\
\text { of Chryse. }\end{array}$ & & & & & IX & & $40^{\circ} \mathrm{N}-25^{\circ} \mathrm{E}$ & $3,13,31,38$ \\
\hline 12. & 222. * & Island of Rhodes. City of Rhodes. & & & & & $\mathrm{X}$ & & $36^{01 / 2} \mathrm{~N}-28^{\circ} \mathrm{E}$ & $3,6,13$ \\
\hline 13. & 138. & $\begin{array}{l}\text { Coasts of Israel and Lebanon. } \\
\text { Between Acre (Ptolemais) and Sur }\end{array}$ & IV & & & & & & & $6,13,35$ \\
\hline 14. & 58. & $\begin{array}{l}\text { Albanian coasts. Durazzo } \\
\text { (Dyrrhachion) }\end{array}$ & II & & & & & & & 4,13 \\
\hline 15. & $23 \pm 3$ & $\begin{array}{l}\text { Egyptian coasts between Alexan- } \\
\text { dria and Pelusium, }\end{array}$ & III & & & & IX & & $38 \cdot 1 / 4 \mathrm{~N}-2201 / 4 \mathrm{E}$ & $6,13,31$ \\
\hline 16. & $46^{*}$ A.D. & South Coasts of Crete. & & & & & VII & & $36^{\circ 1 / 4} \mathrm{~N}-25^{\circ 1 / 4} \mathrm{E}$ & 13,38 \\
\hline
\end{tabular}


cont. CaT.

\begin{tabular}{|c|c|c|c|}
\hline 17. & 62 & South coasts of Crete. Lebena & III \\
\hline 18. & $76^{\star}$ & $\begin{array}{l}\text { Cyprus. Kition, Paphos and Sa- } \\
\text { lamis. }\end{array}$ & \\
\hline 19. & 142 & $\begin{array}{l}\text { Island of Rhodes. Rhodes (Iv), } \\
\text { Islands of Kos, Seriphos and } \\
\text { Syme }\end{array}$ & IV \\
\hline 20. & 262 & South coasts of Asia Minor & IV \\
\hline 21. & 315 & Dead Sea & III \\
\hline 22. & $342^{\star}$ & Cy'prus. Famagusta. & \\
\hline 23. & 344 & $\begin{array}{l}\text { Dardanelles (Hellespontos) and } \\
\text { Niksar (Neocaesaria), Thracian } \\
\text { Coasts }\end{array}$ & IV \\
\hline 24. & 348 & $\begin{array}{l}\text { Syrian coasts. Beirut, Arwad } \\
\text { Islands. }\end{array}$ & III \\
\hline 25. & 362 & Dead Sea, Jordanian coasts. & III \\
\hline 26. & 365 July 21 & $\begin{array}{l}\text { Eastern Mediterranean. Methone, } \\
\text { Epidaurus, Crete, Beeotian coasts, } \\
\text { Adriatic coasts, Epirus, Alexan- } \\
\text { dria, Sicily }\end{array}$ & IV \\
\hline 27. & 447 November & $\begin{array}{l}\text { Sea of Marmara. Marmara Islands } \\
\text { Dardanelles, and the coasts of } \\
\text { Marmara, Constantinople }\end{array}$ & IV- \\
\hline 28. & 450 January & Sea of Marmara, Constantinople. & III \\
\hline 29. & 542 Winter & $\begin{array}{l}\text { Sea of Marmara. Thracian coasts } \\
\text { and Gulf of Edremit }\end{array}$ & IV \\
\hline 30. & 551 Spring & Malian Gulf. Achinos, Tarphe & \\
\hline 31. & 551 * July 7 & Etolia, Nafpaktos & \\
\hline 32. & 551 July 9 & $\begin{array}{l}\text { Syrian coasts. Votrys near Ju- } \\
\text { beil (Byblos), Tarabulus (Tripolis), } \\
\text { Beirut }\end{array}$ & $+\mathrm{III}$ \\
\hline 33. & 551 August 15 & $\begin{array}{l}\text { Southwest coasts of Asia Minor, } \\
\text { Mandalya Bay, Island of Kos, } \\
\text { Sporades Isls }\end{array}$ & IV-- \\
\hline
\end{tabular}

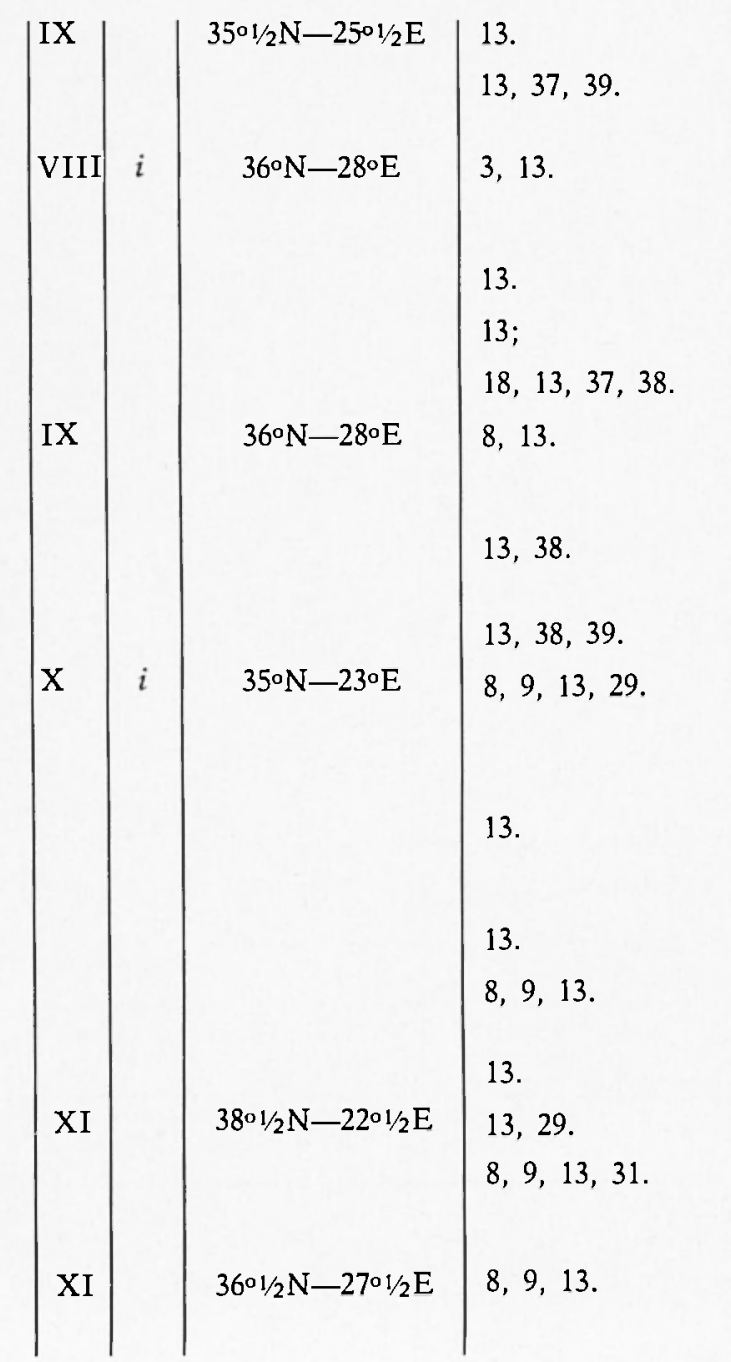

\begin{tabular}{|c|c|c|c|}
\hline 34. & 740 October 26 & $\begin{array}{l}\text { Sea of Marmara, Izmit, Iznik } \\
\text { Lake, Thracian coasts of Marmara }\end{array}$ & III \\
\hline 35. & 746 January 18 & Syrian and Egyptian coasts. & III-- \\
\hline 36. & 792 & $\begin{array}{l}\text { Gulf of Venice, coasts of Istria } \\
\text { and Jugoslavian coasts of the } \\
\text { Adriatic sea }\end{array}$ & IV \\
\hline 37. & 803 December 19 & Gulf of Iskenderun. Masisa coast & III \\
\hline 38. & 859 November & Syrian Coasts, near Samandag & III \\
\hline 39. & $881^{*}$ & $\begin{array}{l}\text { Coasts of Israel and Egypt, from } \\
\text { Aere to Alexandria. }\end{array}$ & \\
\hline 40. & 957 & Caspian Sea & III \\
\hline 41. & 975 October 26 & $\begin{array}{l}\text { Constantinople and Thracian } \\
\text { coasts }\end{array}$ & III \\
\hline 42. & $991 *$ April 5 & Coasts of Syria. & \\
\hline 43. & 1034 January 4 & $\begin{array}{l}\text { Coasts of Lebzanon and Israel } \\
\text { Acre }\end{array}$ & IV \\
\hline 44. & 1039* January & Constantinople. & \\
\hline 45. & $1050^{*}$ & $\begin{array}{l}\text { Grecian Archipelago. Thera (San- } \\
\text { torini). Most probably a misprint } \\
\text { for } 1650\end{array}$ & \\
\hline 46. & 1068 March 18 & $\begin{array}{l}\text { Coasts of Israel at Holots Ashod } \\
\text { and Yavne }\end{array}$ & IV \\
\hline 47. & 1202 May 22 & Syrian coasts, Cyprus, Egypt & IV \\
\hline 48. & 1222 May. Cyprus & Limassol and Paphos & IV \\
\hline 49. & 1273 September & Albanian coasts. Durazzo & III \\
\hline 50. & 1303 August 8 & $\begin{array}{l}\text { Egyptian coasts, Syrian coasts } \\
\text { Crete }\end{array}$ & $v-$ \\
\hline 51. & 1303 December & $\begin{array}{l}\text { Crete, Southwest coasts of Pelo- } \\
\text { ponnesus and Rhodes, Egyptian } \\
\text { coasts and Adriatic Sea }\end{array}$ & IV \\
\hline & 1332 February 12 & Marmara. Con & \\
\hline
\end{tabular}

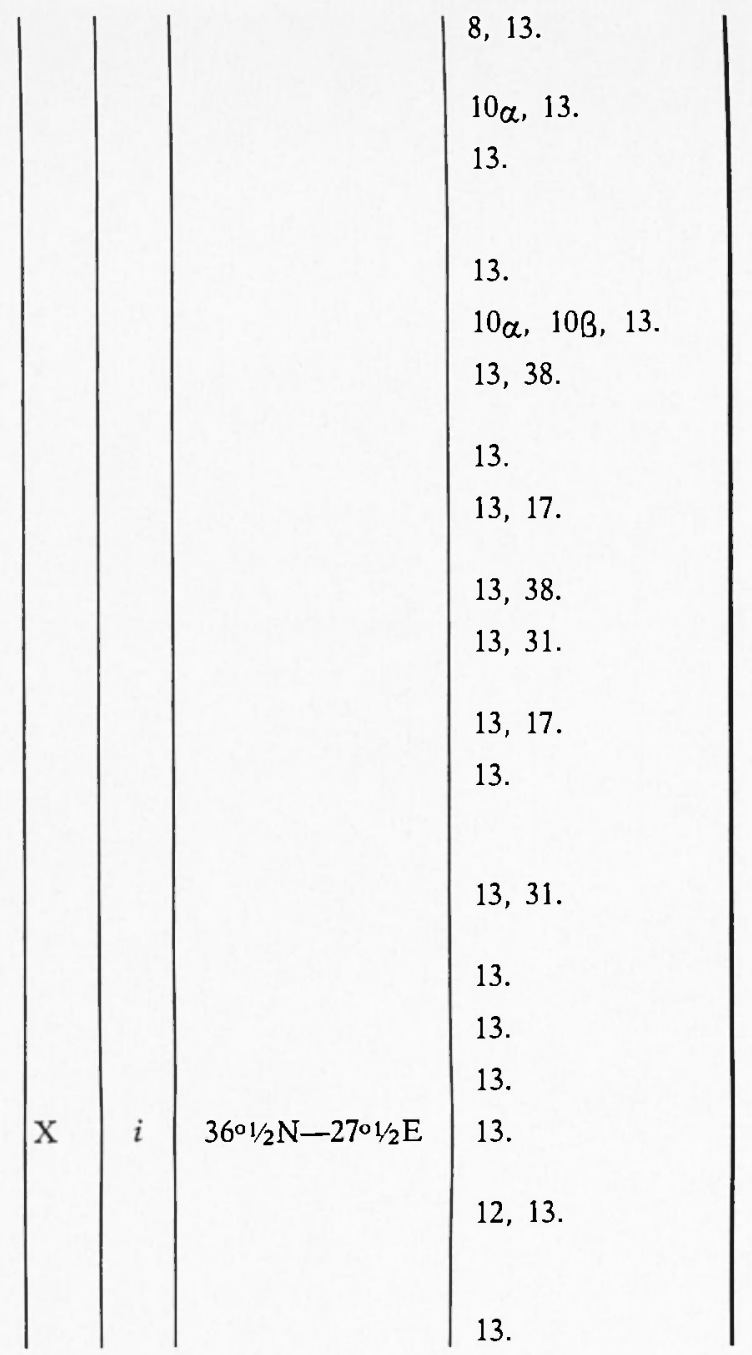


cont. Cat.

\begin{tabular}{|c|c|c|c|c|c|c|c|c|c|}
\hline 53. & 1344 October 14 & Sea of Marmara. Thracian coasts & IV & & | $2000 \mid$ & & & & 13. \\
\hline 54. & 1389 March 20 & $\begin{array}{l}\text { Island of Chios. In the Allatiano } \\
\text { Codex }\end{array}$ & III & & & IX & & $38^{01 / 4} \mathrm{~N}-26^{\circ} 1 / 4 \mathrm{E}$ & 13. \\
\hline 55. & 1403 November 16 & $\begin{array}{l}\text { Syrian coasts, Asia Minor south } \\
\text { coasts }\end{array}$ & III & & & & & & 13. \\
\hline 56. & 1481 May 3 & Rhodes & III & 1,80 & 60 & VIII & & $36^{\circ} \mathrm{N}-28^{\circ} \mathrm{E}$ & 13. \\
\hline 57. & 1489 & $\begin{array}{l}\text { South coasts of Asia Minor. An- } \\
\text { talya }\end{array}$ & III & & & & & & 13. \\
\hline 58. & 1494 July 1 & Crete. Herakleion (Candia) & $\mathrm{II}+$ & & & & & & 13. \\
\hline 59. & 1508 May 29 & South coasts of Crete north coasts & III & & & $\mathrm{X}$ & $i$ & $35^{\circ} 1 / 2 \mathrm{~N}-25^{\circ} 1 / 2 \mathrm{E}$ & 13. \\
\hline 60. & 1509 September 14 & Constantinople & III & & & & & & 13,32 . \\
\hline 61. & 1534 & $\begin{array}{l}\text { Coasts of Israel. Jaffa. Not found } \\
\text { in contemporary writers }\end{array}$ & III & & & & & & 13,38 . \\
\hline 62. & 1546 January 14 & $\begin{array}{l}\text { Coasts of Israel and Lebanon. } \\
\text { From Gaza to Jaffa. }\end{array}$ & III + & & & VII & & $38^{\circ} 1 / 4 \mathrm{~N}-26^{\circ} 1 / 4 \mathrm{E}$ & 13. \\
\hline 63. & 1612 November 8 & North coasts of Crete & IV + & & & VIII & & $35^{\circ} 1 / 2 \mathrm{~N}-25^{\circ} \mathrm{l} / 2 \mathrm{E}$ & 13. \\
\hline 64. & 1622 May 5 & Ionian Island. Zante & & & & $\mathrm{X}$ & & $37^{\circ} 1 / 2 \mathrm{~N}-21^{\circ} \mathrm{E}$ & 13,29 . \\
\hline 65. & 1629 March 9 & Islands of Cythera and Crete & III & & & VIII & & $36^{\circ} \mathrm{N}-25^{\circ} \mathrm{E}$ & 13. \\
\hline 66. & 1633 November 5 & $\begin{array}{l}\text { Zante, South coasts on the pro- } \\
\text { montory of Agios Sostis. }\end{array}$ & III- & & & $\mathrm{X}$ & & $37^{\circ} 1 / 2 \mathrm{~N}-21^{\circ} \mathrm{E}$ & 13,14 . \\
\hline 67. & 1646 April 5 & Constantinople & III & & & & & & 13,31 . \\
\hline 68. & 1650 October 9 & $\begin{array}{l}\text { Thera, west coasts of Patmos, } \\
\text { east coasts, Ios, Sikinos, Kea, } \\
\text { Crete }\end{array}$ & $\mathrm{v}$ & 30 & & VIII & & $36^{\circ} 1 / 2 \mathrm{~N}-25^{\circ} 1 / 2 \mathrm{E}$ & 13. \\
\hline 69. & 1667 April 6 & $\begin{array}{l}\text { Dalmatian coasts. Dubrovnik (Ra- } \\
\text { gusa) }\end{array}$ & III + & & & & & & 13. \\
\hline 70. & 1667 November 30 & Asia Minor. Smyrna & II & & & & & & 13. \\
\hline 71 & $1672^{*}$ & Kiklades. Thera (Santorini) & & & & IX & & $36^{\circ} 1 / 2 \mathrm{~N}-25^{\circ} 1 / 2 \mathrm{E}$ & $13,29$. \\
\hline 72 . & 1672* April & Islet of Stanchio. & & & & VIII & & $40^{\circ} \mathrm{N}-26^{\circ} \mathrm{E}$ & $13,29,31$ \\
\hline & 1688 July 10 & Asia Minor, Smyrna & II & & & & & & $13,31$. \\
\hline
\end{tabular}

\begin{tabular}{|c|c|c|c|}
\hline 74. & 1723 February 21 & Ionian Islands, Leukas. & III \\
\hline 75. & 1732 & Ionian Islands. Corfu & II- \\
\hline 76. & 1748 May 14 & North Peloponnesus. Aigion & III \\
\hline 77. & 1750 September 17 & Adriatic Sea. Fiume & IV \\
\hline 78. & 1752 July 21 & Syrian coasts & III \\
\hline 79. & 1759 October 30 & $\begin{array}{l}\text { Coasts of Israel and Lebanon. } \\
\text { Acre }\end{array}$ & IV- \\
\hline 80. & 1766 May 22 & Constantinople & II \\
\hline 81. & 1791 November 2 & $\begin{array}{l}\text { Ionian Islands. Between Zante } \\
\text { and the mainland }\end{array}$ & III- \\
\hline 82. & 1802 January 4 & Adriatic Sea. Fiume & III \\
\hline 83. & 1804 June 8 & $\begin{array}{l}\text { Northwest coasts of Peloponnesus. } \\
\text { Patras }\end{array}$ & III \\
\hline 84. & 1817 August 23 & Gulf of Corinth. Aigion & III \\
\hline 85. & $1818^{*}$ January & $\begin{array}{l}\text { Athens. Mallet mistakes the event } \\
\text { before mentioned }\end{array}$ & \\
\hline 86. & 1821 January 6 & Gulf of Corinth. Alcyonic Sea & IV \\
\hline 87. & 1823 August 20 & Dalmatian coasts. Dubrovnik & IV - \\
\hline 88. & 1825 January 19 & $\begin{array}{l}\text { Ionian Islands. Leukas, between } \\
\text { the islet of Sessoula and east } \\
\text { coasts of the island }\end{array}$ & III \\
\hline 89. & 1829 May 23 & Constantinople & II \\
\hline 90. & 1833 January 19 & $\begin{array}{l}\text { Albanian coasts. Valona and Sa- } \\
\text { seno Isle }\end{array}$ & IV - \\
\hline 91. & 1835 July 12 & Ionian Islands. Zante & II \\
\hline 92. & 1837 January 1 & $\begin{array}{l}\text { Syrian and Israeli coasts of } \mathrm{Ti}- \\
\text { berias }\end{array}$ & IV \\
\hline 93. & 1843 September 14 & $\begin{array}{l}\text { Dalmatia. Dubrovnik, Gruz (Gra- } \\
\text { vosa) }\end{array}$ & IV \\
\hline & 1844 March 3 & Dalmatia. Dubrovnik & III \\
\hline
\end{tabular}

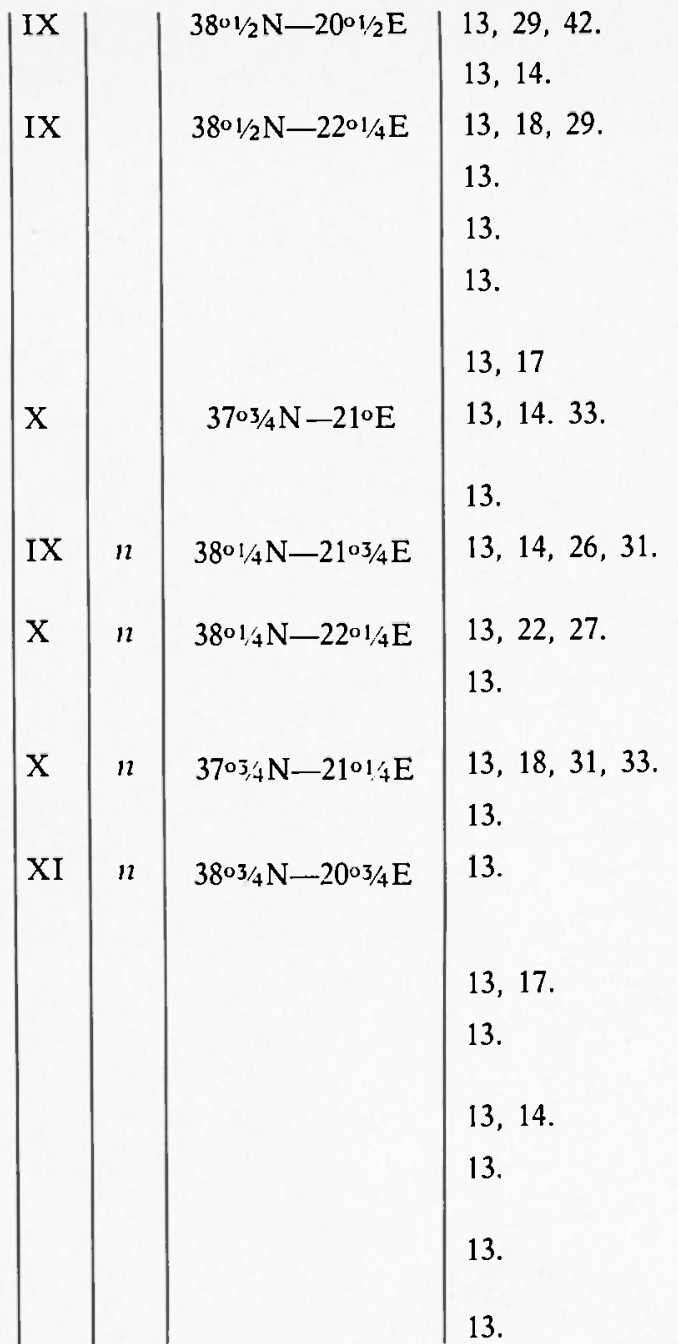


con CAT.

\begin{tabular}{|c|c|c|c|c|c|c|c|c|}
\hline 95. & 1844 March 23 & Dalmatia. Dubrovnik & II & & & & & 13. \\
\hline 96. & 1845 August 16 & Dalmatia. Dubrovnik, Gruz & III & & & & & 13. \\
\hline 97. & 1851 February 28 & Asia Minor. Fetiye & III & 0,60 & IX & $n$ & $36^{\circ 1 / 2} \mathrm{~N}-28^{03 / 4} \mathrm{E}$ & 13,26 \\
\hline 98. & 1851 April 3 & Asia Minor. Fetiye & III & 1,80 & & & & 13. \\
\hline 99. & 1851 May 23 & Khalki Isle, Rhodes & II & & & & & 13. \\
\hline 100. & 1851 October 12 & Albanian, coasts, Valone & III & 0,60 & & & & 13. \\
\hline 101. & 1852 May 12 & Asia Minor, Smyrna & III & & & & & 13. \\
\hline 102. & 1852 September 8 & Asia Minor, Smyrna & III & & & & & 13. \\
\hline 103. & 1853 August 18 & Euboean Gulf & $\mathrm{III}+$ & & IX & $n$ & $38^{\circ 1 / 4} \mathrm{~N}-23^{\circ 1 / 2} \mathrm{E}$ & 13. \\
\hline 104. & 1855 February 13 & Asia Minor, Fetiye & III & & & & & 13. \\
\hline 105. & 1856 November 13 & Eastern Sporades. Chios & $\operatorname{III}+$ & & IX & $n$ & $3801 / 4 \mathrm{~N}-2601 / 4 \mathrm{E}$ & $13,21$. \\
\hline 106. & $1859 *$ October 20 & $\begin{array}{l}\text { Piraeus. Most probably a strong } \\
\text { seiche. }\end{array}$ & II- & & & & & 13. \\
\hline 107. & 1861 December 26 & $\begin{array}{l}\text { Corinth Gulf Northeast coasts } \\
\text { South coasts }\end{array}$ & IV- & 2,10 & $\mathrm{XI}$ & $n$ & $38^{01 / 4} \mathrm{~N}-22^{\circ 1 / 4} \mathrm{E}$ & $13,22,27$ \\
\hline 108. & 1866 January 2 & Albanian coasts. Valona, Himara & IV & & & & & 13. \\
\hline 109. & 1866 January 6 & Albanian coasts. Valona, Narta & III & & & & & 13. \\
\hline 110. & 1866 February 2 & Eastern Sporades, Chios & III & & VIII & $n$ & $38^{\circ 1 / 4} \mathrm{~N}-26^{\circ 1 / 4} \mathrm{E}$ & 13. \\
\hline 111. & 1866 February 6 & $\begin{array}{l}\text { South coasts of Peloponnesus. Is- } \\
\text { land of Kythera }\end{array}$ & IV & 7,80 & VIII & $n$ & $36^{\circ} \mathrm{N}-23^{\circ} \mathrm{E}$ & $13,22,26,27$ \\
\hline 112. & 1866 March 3 & Albanian coasts. Valona & III & & & & & 13. \\
\hline 113. & 1866 March 6 & Albanian coasts. Himara, Kanina & IV & & & & & 13. \\
\hline 114. & 1866 March 13 & Albanian coasts. Himara. Kanina & III & & & & & 13. \\
\hline 115. & 1867 September 20 & $\begin{array}{l}\text { South coasts of Peloponnesus, } \\
\text { Gythion, Crete, Herakleion, Jonian } \\
\text { Islands, Syros, Southeast coasts } \\
\text { of Italy }\end{array}$ & IV & & IX & $n$ & $36^{\circ 1 / 2} \mathrm{~N}-22^{\circ 1 / 4} \mathrm{E}$ & $13,22,27$ \\
\hline 116. & 1869 December 28 & Albanian coasts. Valone & III & & $\mathrm{XI}$ & $n$ & $3803 / 4 \mathrm{~N}-2003 / 4 \mathrm{E}$ & $13,20$. \\
\hline
\end{tabular}

\begin{tabular}{|c|c|}
\hline 117. & 1870 June 24 \\
\hline 118. & 1870 July 29 \\
\hline 119. & 1878 April 19 \\
\hline 120. & 1883 June 27 \\
\hline 121. & 1886 August 27 \\
\hline 122. & 1887 October 4 \\
\hline 123. & 1893 February 9 \\
\hline 124. & 1893 June 14 \\
\hline 125. & 1894 April 27 \\
\hline 126. & 1894 July 10 \\
\hline 127. & 1897 December \\
\hline 128. & 1898 Decembczr 3 \\
\hline 129. & 1899 January 22 \\
\hline 130. & 1902 July 5 \\
\hline 131. & 1908 December 28 \\
\hline 132. & 1914 November 27 \\
\hline 133. & 1915 August 7 \\
\hline & 1920 December 18 \\
\hline & 1928 March 31 \\
\hline
\end{tabular}

\begin{tabular}{|c|c|c|}
\hline Alexandria & III & \\
\hline Adriatic Sea. Island of Vis (Lissa) & III & \\
\hline Sea of Marmara. Izmid & III & \\
\hline Ionian Island. Corfu & III & \\
\hline $\begin{array}{l}\text { South coasts of Peloponnesus. } \\
\text { Messenia, Pylos; Asia Minor, } \\
\text { Smyrna }\end{array}$ & III & \\
\hline $\begin{array}{l}\text { Gulf of Corinth. South coasts, } \\
\text { Xylokastro, between Xylokastro } \\
\text { and Sykia }\end{array}$ & III & \\
\hline $\begin{array}{l}\text { Sporades Islands. North coast of } \\
\text { Samotrace, Alexandroupolis } \\
\text { Thracian coasts }\end{array}$ & $\mathrm{III}+$ & 0,90 \\
\hline Albanian coasts. Valona, Himara & III & \\
\hline $\begin{array}{l}\text { Euboean Gulf. Atalanta Bay, West } \\
\text { and Northwest coasts of Euboean. } \\
\text { Gulf }\end{array}$ & IV & 3,00 \\
\hline Constantinople & III & \\
\hline Ionian Island. Zante & $\mathrm{II}-$ & \\
\hline Ionian Island. Zante & $\mathrm{II}+$ & \\
\hline Messenia, Kyparissia, Marathos & III & \\
\hline Salonika & $\mathrm{II}-$ & \\
\hline $\begin{array}{l}\text { Lybian Sea. } 90 \text { miles north of } \\
\text { Alexandria, Egyptian coasts }\end{array}$ & $\mathrm{v}$ & \\
\hline Ionian Islands. Leukas & $\mathrm{IV}+$ & 3,30 \\
\hline $\begin{array}{l}\text { Ionian Islands. Between Cephalo- } \\
\text { nia and Leukas }\end{array}$ & III + & \\
\hline $\begin{array}{l}\text { Albanian coasts. Valone, Saseno } \\
\text { Asia Minor. Smyrna }\end{array}$ & $\begin{array}{l}\mathrm{V}- \\
\mathrm{II}\end{array}$ & \\
\hline
\end{tabular}

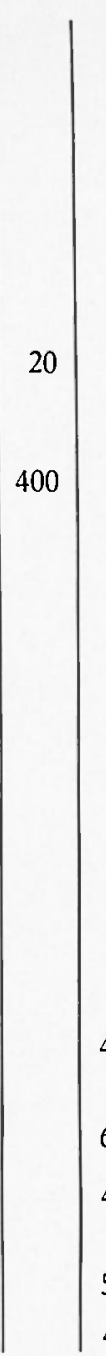


cont. CAT.

\begin{tabular}{|c|c|c|c|c|c|c|c|c|c|c|}
\hline 136. & 1928 April 23-25 & $\begin{array}{l}\text { Grecian Archipelago; Piraeus, } \\
\text { Chalkis, Nauplion, Alexandroupo- } \\
\text { lis, Crete, Chania, Karystos }\end{array}$ & $\mathrm{III}+$ & $2,10 \mid$ & & 4,6 & |VI & $n$ & $42,4^{\circ} \mathrm{N}-25,7^{\circ} \mathrm{E}$ & 13. \\
\hline 137. & 1928 May 3 & Eastern Greece. Strymonic Gulf & II & & & 4,3 & VII & $n$ & $40,8^{\circ} \mathrm{N}-26,8^{\circ} \mathrm{E}$ & 13. \\
\hline 138. & 1932 September 26 & Gulf of Hierissos. Chalcidice & $\mathrm{II}+$ & & & 6,9 & $\mathrm{X}$ & $n$ & $4001 / 2 \mathrm{~N}-23^{03 / 4} \mathrm{E}$ & 13. \\
\hline 139. & 1947 October 6 & $\begin{array}{l}\text { South Peloponnesus. Methone in } \\
\text { Messenia }\end{array}$ & II + & & & 6,9 & IX & 28 & $36,9 \circ \mathrm{N}-22^{\circ} \mathrm{E}$ & $13,19,27$ \\
\hline 140. & 1948 February 9 & Dodecanese. Island of Karpathos & IV & & 1000 & 7,1 & IX & 40 & $35^{\circ} 1 / 2 \mathrm{~N}-27^{\circ} \mathrm{E}$ & $13,22,26,27$. \\
\hline 141. & 1948 April 22 & Ionian Islands. Leukas & IV- & 0,90 & & 6,4 & $\mathrm{X}$ & $n$ & $38 \circ 1 / 2 \mathrm{~N}-2001 / 4 \mathrm{E}$ & $13,22,26,27$. \\
\hline 142. & 1949: February 9 & Dodecanese. Island of Karparhos & & & & & & $n$ & & 13,30 . \\
\hline 143. & 1956 July 9 & $\begin{array}{l}\text { Grecian Archipelago. Amorgos, } \\
\text { Astipalaea, Pholegandros, Patmos, } \\
\text { Kalimnos, Crete, Tinos }\end{array}$ & $\mathrm{v}$ & 30 & & 7,8 & IX & 20; & $36,9^{\circ} \mathrm{N}-26^{\circ} \mathrm{E}$ & $13,24,26,27$. \\
\hline 144. & 1956 November 2 & Magnessia. Volos & $\mathrm{II}+$ & 1,20 & & $3 / 4$ & VII & $n$ & $39 \circ 1 / 2 \mathrm{~N}-23^{\circ} \mathrm{E}$ & $13,26,28$ \\
\hline 145. & 1959* February 23 & $\begin{array}{l}\text { North and west coasts of the } \\
\text { Grecian Archipelago. Salonika, Sa- } \\
\text { lamis, Leros, Crete. No earthquake } \\
\text { shock was recorded. Most pro- } \\
\text { bably a seiche. }\end{array}$ & III & 0,90 & & & & & & 13. \\
\hline 146. & 1961* May 23 & $\begin{array}{l}\text { Asia Minor. Sea of Marmara, } \\
\text { Smyrna. }\end{array}$ & & & & 6,5 & VII & $n$ & & 13. \\
\hline 147. & $1961 *$ June 6 & $\begin{array}{l}\text { Grecian Archipelago. Crete, Volos, } \\
\text { Leros. Probably strong seiche }\end{array}$ & II & 0,90 & & & & & & 13. \\
\hline 148. & 1963 February 7 & $\begin{array}{l}\text { Gulf of Korinth. Possidonia, } \\
\text { Patras. }\end{array}$ & & & & & & & & 13,44 . \\
\hline 149. & 1965 July 6 & Gulf of Korinth. Itea bay. & $\mathrm{II}+$ & & & 6,9 & VIII & & $38,4 \circ \mathrm{N}-22,3 \circ \mathrm{E}$ & 13. \\
\hline 150. & 1968 February 19 & $\begin{array}{l}\text { Island St. Eustratios, Lemnos, } \\
\text { Lesvos, Euboea. }\end{array}$ & $\mathrm{II}+$ & 1,20 & & 6,7 & IX & 31 & $39,5 \mathrm{~N}-23,8^{\circ} \mathrm{E}$ & 13. \\
\hline $151 .^{*}$ & 1978 June 20 & $\begin{array}{l}\text { Salonica, Strymonic Gulf. } \\
\text { Most probably a strong seiche. }\end{array}$ & II- & - & - & 6,5 & $=$ & 16 & $40,75^{\circ} \mathrm{N}-23,26^{\circ} \mathrm{E}$ & 43,45 . \\
\hline $152 . *$ & 1979 May 15 & $\begin{array}{l}\text { South Coasts of Crete. Most pro- } \\
\text { bably a strong seiche. }\end{array}$ & II- & - & - & 5,8 & - & 58 & $34,62^{\circ} \mathrm{N}-24,08^{\circ} \mathrm{E}$ & 43,45 . \\
\hline
\end{tabular}




\section{ACKNOWLEDGEMENTS}

I have been much assisted by Professor N. Ambraseys of the Imperial College - University of London - to whom I am intebted.

Without his invaluable help this work could not have been carried out. 


\section{REFERENCES}

\section{CLASSIC SOURCES}

Aristotle, (a) Meteorologika. (b) De mundo.

Herodotos, Historiae.

Pausanias, Graeciae descriptio.

Plutarchis, Vitae.

Seneca, Question. Natural.

Strabo, Geographica.

TIIUCydides, Historiae.

CEDrinos, Cedrinos Georgios compendium historiarum.

Matalas, Chronographia Ioannis Malaiae.

\section{ARABIC, SOURCES}

JIRGIS EL-MACIN IGN EL-AMID, (a) in Th. Erpenius's Latin translation: Historia Suraccua-Arcbice olim extrata a Georgio Elmakina et Latina redditc opera ac studio. Lugduni Batavorum, 1625. (b) in P. Vattier's translation: L'histoire mahométane ou les quaranteneuf chalifes, Paris 1657.

\section{CONTEMPORAR'Y SOURCES}

Ambraseys N., 1960 - The seismic sea-wave of July 1956 in the Greak Archipelago. "Journ. Geoph. Research », 65, Nr. 4, p. 1257.

Ambraseys N., 1961 - On the seismicity of Southwest Area. "Revue pour l'Etude de Calamités ", Nr. 37, Geneve.

Ambraseys N., 1962 - Data for the investigation of the seismic seawaves in the Eastern Mediterranean. "Bull. Seism. Soc. Am.,", 52, No. 4, pp. 895-913, October. 
Barbiani D., Barbiani B., 1864 - Mémoire sur les tremblements de terre dans l'ile de Zante. Mémoires de l'Académie de Dijon, Sci. Section, 11, Dijon.

Berneertz, Vid., 1616 - Terrae motus, das ist gründliches Bericht von den Erdbiden, etc. Nürnberg.

BRASLAFSKI I., 1938 - The 1546 earthquake and the arresting of the flow of the Jordan river. "Zioni" 3, New Series, Jerusalem, p. 330, (in Hebrew).

Dück J., 1904 - Die Erdbebeben von Konstantinopel. "Die Erdbebenwarte ", col. 3, Laibach.

Galanopoulos A., 1938 - La sismicité de l'Achaie et des régions voisines de Missolonghi et de Naupacte. "Gerl. Beitr. Geophys.", 53, Leipzig.

Galanopoulos A., 1949 - The Koroni, Messina, earthquake of October 6, 1947. "Bull. Seism. Soc. Amer.", 39, Nr. 1.

Galanopoulos A., 1952 - Die Seismizität der Insel Leukas; Allgemeine historisque Uebericht. "Gerl. Beitr. Geophys.", 62, Nr. 4, Leipzig.

Galanopoulus A., 1953 - Katalog der Erdbeben in Griechenland für die Zeit von 1879 bis 1892. "Annales Géologiques" des Pays Helléniques, 5, Athens.

Galanopoulus A., 1955 - Earthquake geography of Greece. "Annales Géologiques " des Pays Helléniques, 6, Athens in Greek).

Galanopoulos A., 1957 - Zur Bestimmung des Alters der Santorin-Kaldera.

"Neues Jb. Geol. Paläontol. Mh. », 9, p. 419, Stuttgart.

Galanopoulos A, 1957 - The seismic sea-wave of 9th July 1956. "Praktika Akademias Athenon ", 32, p. 90, Athens (in Greek).

Galanopoulos A., 1958 - Zur Bestimmung des Alters der Santorin-Kaldera. Annales Géologiques " des Pays Helléniques, 9, p. 185, Athens.

Galanopoulos A., 1960 - A catalogue of shocks in Greece with $I>V I$ or $M>5$ for the years 1801 to 1958. Publications, Seismological Laboratory, University of Athens, Athens.

Galanopoulos A., 1960 - Tsunamis observed on the coasts of Greece from antiquity to present times. "Annali di Geofisica ", 13, 3-4, Rome.

Galanopoulos A., 1960 - On the origin of the deluge of Deukalion and the Myth of Atlantis. "Archaelogiki Etairia Athenon", 3-EE, p. 226, Athens, (in Greek).

Galanopoulos A., 1961 - A catalogue of shock in Greece with $I>V I I$ for the years prior to 1800. Publications, Seismological Laboratory, University of Athens, Athens. 
Calanopoylos A., Bacon E., 1969 - Atlantis, The truth behind the legend. Thomas Nelson and Sons LTD London.

HorF, K. von 1841, Chronik der Erdbeben und Vulkan-Ausbrïche, mit vorausgehender Abhandlung iiber die Natur dieser Erscheinungen. Gesch. Ueberlieferung nachgew. natürl. Veränder. Erdoberfläche, Parts 4 and 5, Gotha.

Lycosthenes, C.W., 1557 - Prodigiorum ac ostentorum chronicon. Ed. Vid., Basel.

MALLET R., 1850-1856 - Report on the fact of earthquake phaenomena. British Association for the Advancement of Science, Part 1, 1850 pp. 89, Part. 2, 1852 p. 212, Part 3, 1854 pp. 326, Part 4, 1859 pp. 136. Published by J. Taylor.

Marinatos S, 1934 - Amnisos die Hafenstadt des Minos. "Forsh. und Fortschr, ", 10, Nr. 28, p. 342.

Marinatos S., 1939 - The volcanic destruction of Minoan Crete. "Antiquity", 13, Nr. 52, p. 246, Gloucester.

Marinos G., Melidonis N., 1971 - On the strenght of seaquakes (Tsunamis) during the prehistoric eruptions of Santorin. Institute of Geology and subsurface Research. From the acta of the Ist International Scientific Congress on the volcano of Thera, Athens 1969.

Oberhummer E., 1903 - Die Insel Cypern. Eine Landeskunde auf Historischer Grundlage. Ed. Th. Ackermann, München.

SIEBERG A., 1932 - Untersuchungen iiber Erdbeben und Bruchschollenban im östlichen Mittelmeergebiet. "Denkschriften der Medizinish-Naturwissenschaftlichen Gesellschaft zu Jena ", vol. 2, Jena.

Sieberg A., 1932 - Die Erdbeben. In B. Gutenberg's Handbuch der Geophysik, 4, Berlin, Ed. G. Borntraeger.

TECHENER, vid., 1861 - Le grand tremblement et éspouvantable ruine qui est advenue en la cité de Jérousalem et par toute la province d'icelle etc... "Bullétin du Bibliophile», Nr. January, Ed. at St. Sebastien, Paris.

Tholozan J.D., 1879 - Sur les tremblements de la terre qui ont eul lieu en l'Orient du $V I I^{e}$ au XVII $I^{e}$ siècle. Comptes Rendus de l'Académie des Sciences, 83, p. 1063, Paris.

Tsitselis E., 1961 - Chephalinian mélanges. Ed. vid., 3, Athens (in Greek).

Seismological Institute Bulletin, Athens.

Annales Géologiques des Pays Hélleniques.

Periodical, Publications, Press reports. 